\title{
NoMoS: Beyond the Standard Model Physics in Neutron Decay
}

\author{
Gertrud Konrad ${ }^{* a b}$ \\ ${ }^{a}$ Stefan-Meyer-Institute for Subatomic Physics, Austrian Academy of Sciences, \\ Boltzmanngasse 3, 1090 Vienna, Austria \\ ${ }^{b}$ Atominstitut, Technische Universität Wien, \\ Stadionallee 2, 1020 Wien, Austria \\ E-mail: Gertrud.Konradeoeaw.ac.at
}

The newly established New Frontiers Group 'NoMoS: Beyond the Standard Model Physics in Neutron Decay' of the Austrian Academy of Sciences aims to search for traces of new physics in neutron beta decay with novel experimental techniques. The NoMoS group is hosted at the Stefan-Meyer-Institute for Subatomic Physics in Vienna.

Precision measurements in neutron decay allow searching for physics beyond the Standard Model of particle physics. An accuracy of $10^{-4}$ in the observables corresponds to energy scales of $1-100 \mathrm{TeV}$ for new particles and interactions. In order to achieve this accuracy, a new experimental technique is developed: $\mathbf{R} \times \mathbf{B}$ spectroscopy. An $\mathbf{R} \times \mathbf{B}$ spectrometer measures the momentum of charged particles by their drift in a circular magnetic field. This new, accurate method of spectroscopy will be applied to determine several correlation coefficients in neutron decay. The construction of the first $\mathbf{R} \times \mathbf{B}$ spectrometer, NoMoS, is part of the New Frontiers Group.

For measurements at ultimate statistics, the $\mathbf{R} \times \mathbf{B}$ spectrometer will be installed at PERC, a new facility at the FRM II in Garching/Germany that collects electrons and protons from a large neutron decay volume. A final goal is to measure or to set limits on the Fierz interference term. This term is forbidden in the Standard Model and has not yet been measured with neutrons. A non-zero value would indicate that yet unknown charged Higgs bosons, sleptons, or leptoquarks were exchanged instead of the Standard Model $W$ boson. Besides the physics motivation, the measurement concept and physics programme of NoMoS are presented in this paper.

PACS: 13.30.Ce, 14.20.Dh, 23.40.Bw, 29.30.Aj, 29.30.Ep, 29.40.Gx

The European Physical Society Conference on High Energy Physics

22-29 July 2015

Vienna, Austria

${ }^{*}$ Speaker. 


\section{Introduction}

In 1933, A. Einstein gave his Herbert Spencer Lecture 'On the Methods of Theoretical Physics' at the University of Oxford. He proclaimed that 'our experience hitherto justifies us in trusting that nature is the realization of the simplest that is mathematically conceivable' [1]. At present, the Standard Model (SM) of particle physics is a theory that describes all know particles and three of the four fundamental forces of nature extremely well. However, experimental facts as well as theoretical arguments show that the SM cannot be the final theory of particles and interactions. Thus, information about the physics beyond the SM is desperately searched. This information can be found by creating yet unknown particles at accelerators of highest energy (energy frontier; LHC) or by looking for traces that new particles leave in known processes (precision frontier).

NoMoS, the newly established New Frontiers Group of the Austrian Academy of Sciences (ÖAW), is part of the precision frontier. The group aims to study the laws of nature (in Greek: nomos tes physeos) through precision measurements in neutron beta decay. In the SM, neutron decay is well described by two specific parameters only. It is studied by measuring different correlations between decay products and possibly the neutron spin (see Fig. 1). In the past, such precision measurements in nuclear and neutron beta decay have

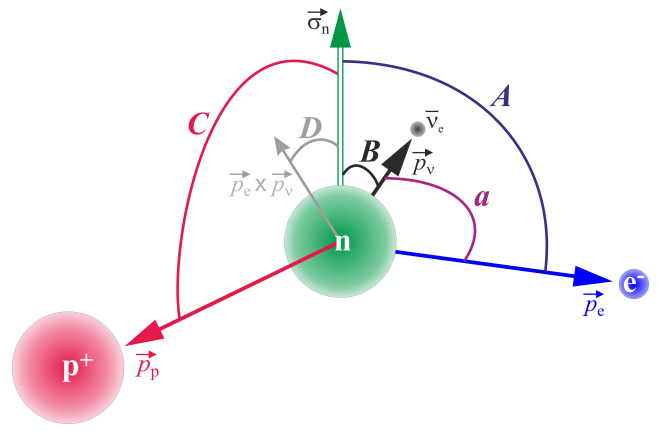

Figure 1: Illustration of the angular correlation coefficients $a, A, B, C$, and $D$ in neutron beta decay. contributed considerably to our understanding of the SM. Today, they are used to search for new physics $[2,3,4]$. The information obtained from such measurements is complementary to direct searches for new physics at high energies with colliders. Low-energy searches with $10^{-4}$ sensitivity would probe energy scales of $1-100 \mathrm{TeV}$ [5], far above the production threshold at the LHC.

\section{State of the art}

The most precise measurements of correlation coefficients in neutron decay have reached accuracies of a few times $10^{-3}$ for asymmetries $[6,7,8]$. The parameters describing proton and electron spectra are known much less precisely: the correlation coefficient $a$ is known with $5 \%$ accuracy $[9,10]$, and the Fierz interference term $b$ and the weak magnetism form factor $f_{2}$ have not been resolved in neutron decay yet. In order to improve the precision of neutron decay data, several new instruments have been built or are presently under construction world-wide: aSPECT, PERKEO III, aCORN, Nab, UCNA, UCNb, UCNB, and PERC (see Sect. 3.3). See [2, 3, 4] for recent reviews.

Currently, the most stringent limits on scalar $(\mathrm{S})$ and tensor $(\mathrm{T})$ couplings arise from the determination of the Fierz term in superallowed Fermi beta decays and the radiative pion decay $\pi^{+} \rightarrow \mathrm{e}^{+} v_{\mathrm{e}} \gamma$, respectively. The next best limits on $\mathrm{T}$ couplings come from nuclear beta decays. Groups from Crakow and Leuven have recently developed two new and compact beta spectrometers to determine the Fierz term and the weak magnetism form factor from nuclear beta decays [11] (see also [12]). Good probe nuclei are ${ }^{45} \mathrm{Ca}$ and ${ }^{114} \mathrm{In}$, respectively. However, searches in nuclear 
beta decay are limited by theoretical uncertainties related to nuclear structure respectively recoilorder corrections. At the energy frontier, bounds on $\mathrm{S}$ and $\mathrm{T}$ couplings from the LHC channels $\mathrm{pp} \rightarrow \mathrm{e}+\mathrm{E}_{\mathrm{T}}+\mathrm{X}$ and $\mathrm{pp} \rightarrow \mathrm{e}^{+} \mathrm{e}^{-}+\mathrm{X}$ are on the (sub-)percent-level. See [5] for a recent review.

\section{3. $\mathbf{R} \times$ B Spectroscopy}

$\mathbf{R} \times \mathbf{B}$ spectroscopy is a new, accurate method of momentum spectroscopy that uses the $\mathbf{R} \times \mathbf{B}$ drift effect to disperse charged particles in a magnetic field. An analogous idea has been mentioned by V.M. Lobashev to measure the mass of the electron-antineutrino [13]. His design of a differential spectrometer is based on a MAC-E-filter [14] with an $\mathbf{R} \times \mathbf{B}$ drift.

\section{1 $\mathbf{R} \times \mathbf{B}$ Spectrometer}

The $\mathbf{R} \times \mathbf{B}$ spectrometer [15] schematically shown in Fig. 2 measures the momentumdependent drift of charged neutron decay products in a curved magnetic field. This allows the simultaneous detection of the full spectrum of electrons and protons in a spatial-resolving detector which circumvents limitations due to non-linearity and backscattering in 'standard' energy-resolving detectors and due to flux variations in energy-selecting detectors. For more details on the preliminary design see [15].

The first $\mathbf{R} \times \mathbf{B}$ spectrometer, NoMoS (short for: Neutron decay prOducts MOmentum Spectrometer), is presently under construction at the Technische Universität (TU) and the Stefan-Meyer-Institute (SMI) Wien.

First measurements with neutrons are planned at the Institut-Laue-Langevin (ILL) in Grenoble/ France. These measurements will be the first application of magnetic spectroscopy for precision measurements in neutron decay.

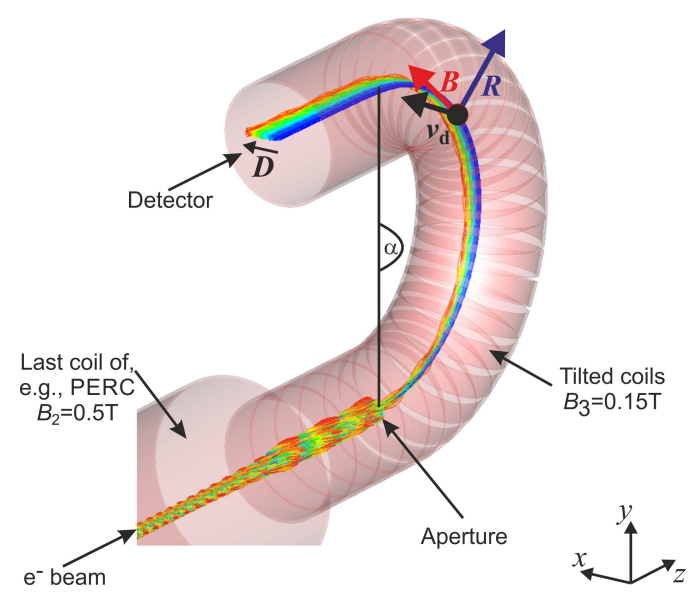

Figure 2: Scheme of the $\mathbf{R} \times \mathbf{B}$ spectrometer coupled to PERC (see Sect. 3.3): Decay electrons are guided by the strong magnetic field towards the half toroid. On their way through the tilted coils, they drift sideways according to their momenta from 0 (blue) to $1.19 \mathrm{MeV} / \mathrm{c}$ (red). After the tilted coils, the detector measures their drift. The magnet coils are drawn in pink. An aperture directly in front of the tilted coils serves to define the dimensions of the incident electron beam. For details see [15].

\subsection{Detector and Calibration}

For the measurement of the momentum-dependent drift in the $\mathbf{R} \times \mathbf{B}$ spectrometer, a spatialresolving detector for low-energy electrons $\left(E_{\mathrm{e}}<780 \mathrm{keV}\right)$ and protons $\left(E_{\mathrm{p}}<750 \mathrm{eV}\right.$, about $15 \mathrm{keV}$ after post-acceleration) is needed. The requirements on this detector and its readout are rather stringent. The detector geometry will be based on validated concepts but tailored to the specific requirements of the $\mathbf{R} \times \mathbf{B}$ spectrometer. This concerns in particular the use of a thick wafer to stop electrons of full energy and a spatial resolution of $1 \mathrm{~mm}$ in one direction. Such a detector is also of great interest for other precision measurements in neutron decay. 
For precision measurements with the $\mathbf{R} \times \mathbf{B}$ spectrometer, the magnetic field, the transfer function, and the detector performance have to be measured and monitored precisely. The following calibration techniques are planned: nuclear magnetic resonance (NMR) magnetometers, standard conversion-electron calibration sources, a compact in-situ proton source, and a source of moderated positrons. The latter two sources are also of high interest for other proton spectrometers.

\subsection{Facility PERC}

PERC (short for: Proton and Electron Radiation Channel) is a special new concept: for the first time charged neutron decay products are collected from a neutron guide and transported by a strong magnetic field to a secondary spectrometer. On their way, the transmitted phase space is selected precisely using a magnetic filter. PERC exploits the full flux of neutrons in a neutron guide and delivers a clean and bright beam of charged neutron decay products [16]. Its preliminary design is schematically shown in Fig. 3. For more details on the new facility PERC see [17].

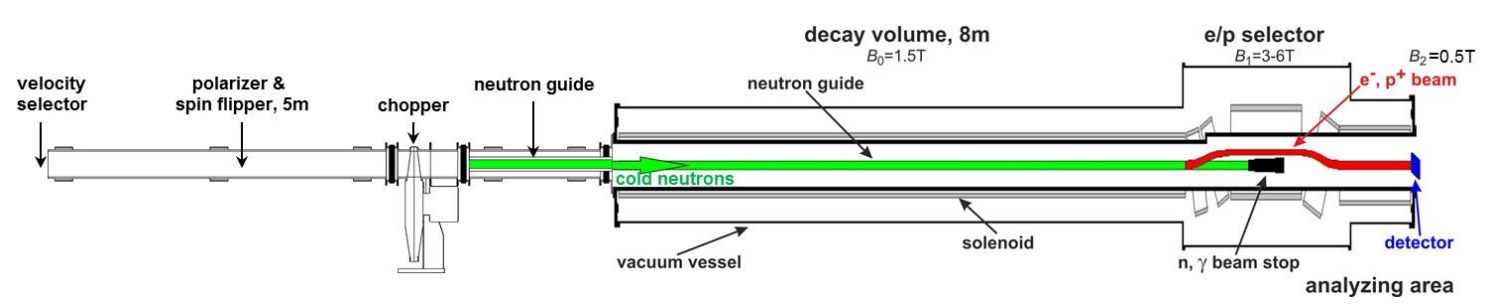

Figure 3: Scheme of the facility PERC: Cold neutrons (green) pass through the decay volume where only a small fraction decays. The decay products (red) are guided by the strong magnetic field towards the detector (blue) or a secondary spectrometer (not shown here). The superconducting coils are drawn in gray. The equipment for neutron beam preparation is located in front of the instrument. For details see [16, 17].

The $\mathbf{R} \times \mathbf{B}$ spectrometer can be coupled adiabatically to the end of PERC. This unique combination of accurate spectroscopy and a clean and bright beam of charged neutron decay products will open the door to reach $10^{-4}$ sensitivity in neutron decay.

\section{NoMoS Physics Programme}

Besides the construction of the $\mathbf{R} \times \mathbf{B}$ spectrometer, the NoMoS group has a comprehensive physics programme to study the structure and nature of weak interactions as well to search for new physics beyond the SM in neutron decay.

\subsection{Research focus}

The following measurements are planned together with further PERC Collaboration members:

- The weak magnetism form factor $f_{2}$. Its value is predicted by the conserved vector current (CVC) hypothesis. But, so far, the value $f_{2}=\left(\kappa_{\mathrm{p}}-\kappa_{\mathrm{n}}\right) / 2 \approx 1.8529$ is tested only to $\mathscr{O}(10 \%)$ in $A=12$ system [18]. The theoretical uncertainties are large.

- The Fierz interference term $b$ (see Fig. 4 (a)). This term is predicted to be equal to zero in the SM and has not yet been resolved in neutron decay. A non-zero value would indicate the existence of S or T interactions. S or T couplings are expected if yet unknown charged Higgs 


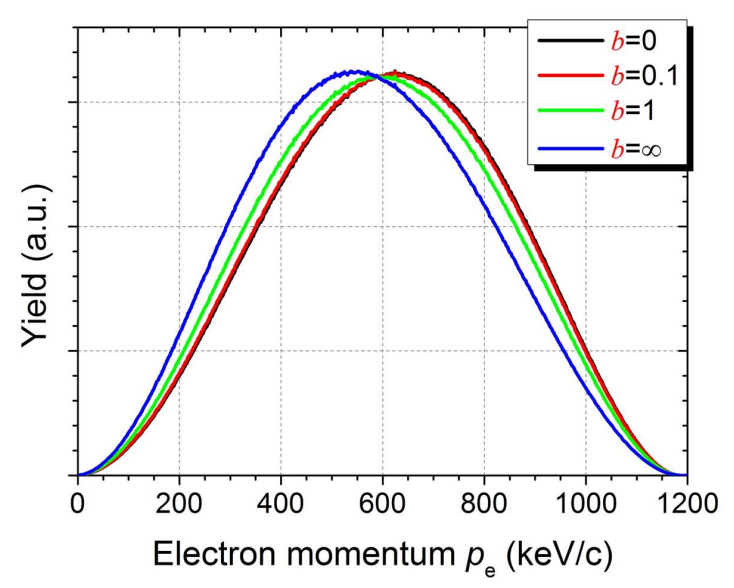

(a) Electron momentum

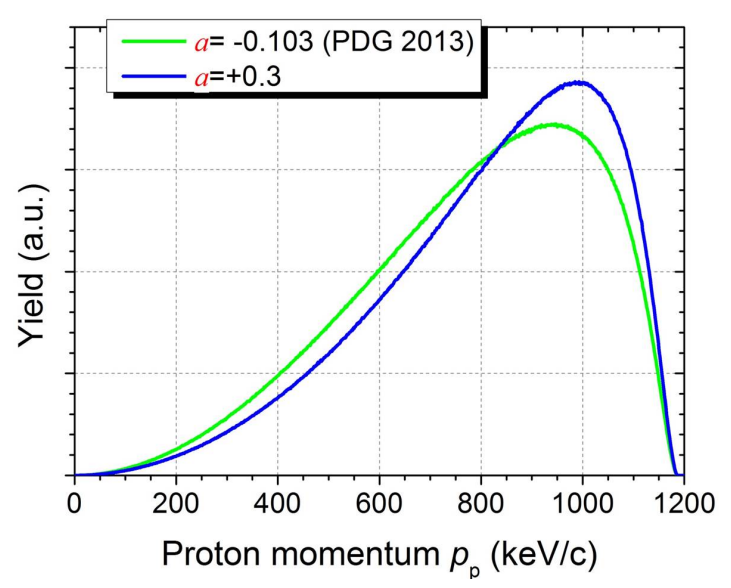

(b) Proton momentum

Figure 4: The theoretical (left) electron and (right) proton momentum spectra in neutron beta decay, respectively. Left: The black line is the prediction from the Standard Model (SM) with $b=0$. The comparison between the black and the other lines shows the influence of the Fierz interference term $b$ on the electron momentum spectrum. Right: The green line is the prediction from the SM with the recommended value for $a=-0.103$, the blue line shows how a deviation from that $(a=+0.3)$ would look like.

bosons, sleptons, or leptoquarks were exchanged instead of the SM $W$ boson. A measurement of or limit on the Fierz term in neutron decay with $10^{-4}$ absolute sensitivity is more sensitive than searches for these couplings at the LHC at full luminosity and energy [5].

- The neutrino-electron correlation coefficient $a$ (see Fig. 4 (b)).

- The beta asymmetry parameter $A$.

- Oscillatory, sidereal effects in the case of Lorentz invariance violation [19].

The correlation coefficients $a$ and $A$ can be used to derive the SM parameters $\lambda$ (ratio of axial vector to vector coupling constant) and $V_{\text {ud }}$ (upper left element of the CKM quark mixing matrix; calculated from $\lambda$ and the neutron lifetime). Interactions beyond the SM, such as e.g. supersymmetry, can be best constrained from tests of the unitarity of the CKM matrix. Furthermore, $\lambda$ serves as input for predictions of solar neutrino fluxes, primordial abundances of light elements, neutron star formation, or for the calculation of the detection efficiency of neutrino detectors.

The goal of NoMoS is electron and proton spectroscopy on the $10^{-4}$ - respectively $10^{-3}$-level.

\subsection{Theoretical prerequisite}

Searches for new physics with $10^{-4}$ sensitivity need adequate input from theory. The following analyses are planned by M. Pitschmann (TU Wien) and co-workers:

- The extension of the existing analysis of correlation coefficients $a, A, B, C$, and $D$, calculated to order $10^{-3}$ within the SM [20], to order $10^{-5}$.

- The completion of the analysis of non-standard correlation coefficients $N, G, R, Q$, and $L$ to order $10^{-3}$ within the SM.

- The most precise possible evaluation of the $\mathrm{S}$ and $\mathrm{T}$ form factors $g_{\mathrm{S}}$ and $g_{\mathrm{T}}$, respectively, within the SM and interactions beyond [21]. 


\section{Summary}

The newly established New Frontiers Group 'NoMoS' of the ÖAW aims to search for new 'physics beyond the SM in neutron decay'. Searches with $10^{-4}$ sensitivity will have unmatched constraining potential, even at full LHC luminosity and energy. In order to explore this precision frontier, a novel experimental technique is developed: $\mathbf{R} \times \mathbf{B}$ spectroscopy. As part of the New Frontiers Group, the first $\mathbf{R} \times \mathbf{B}$ spectrometer is presently under construction at TU and SMI Wien.

\section{Acknowledgments}

The author would like to thank X. Wang, H. Abele, M. Pitschmann (all three from the TU Wien), E. Widmann (SMI Wien) and T. Soldner (ILL) for their support and contributions. This work is supported by the ÖAW within the New Frontiers Groups Programme NFP 2013/09, the German Research Foundation and the Austrian Science Fund as part of the Priority Programme 1491 under contracts No. I 534-N20 and AB 128 5-2, the ILL, and the TU and SMI Wien.

\section{References}

[1] A. Einstein, in: The Herbert Spencer Lecture for 1933, Clarendon Press, Oxford 1933.

[2] H. Abele, Prog. Part. Nucl. Phys. 60 (2008) 1.

[3] D. Dubbers and M.G. Schmidt, Rev. Mod. Phys. 83 (2011) 1111.

[4] B.R. Holstein et al., Focus Section: The precision frontier in semileptonic weak interactions, J. Phys. G 41(11) (2014), articles 114001 - 114007.

[5] V. Cirigliano, S. Gardner, and B.R. Holstein, Prog. Part. Nucl. Phys. 71 (2013) 93.

[6] A.P. Serebrov et al., JETP 86 (1998) 1074.

[7] M. Schumann et al., Phys. Rev. Lett. 99 (2007) 191803.

[8] D. Mund et al., Phys. Rev. Lett. 110 (2013) 172502.

[9] C. Stratowa et al., Phys. Rev. D 18 (1978) 3970.

[10] J. Byrne et al., J. Phys. G 28 (2002) 1325.

[11] N. Severijns, J. Phys. G: Nucl. Part. Phys. 41 (2014) 114006, and references therein.

[12] H. Abele et al., Phys. Lett. B 316 (1993) 26.

[13] V.M. Lobashev, Prog. Part. Nucl. Phys. 40 (1998) 337.

[14] A. Picard et al., Nucl. Instrum. and Meth. B 63 (1992) 345.

[15] X. Wang, G. Konrad, and H. Abele, Nucl. Instrum. and Meth. A 701 (2013) 254 .

[16] D. Dubbers et al., Nucl. Instrum. and Meth. A 596 (2008) 238.

[17] G. Konrad et al. (The PERC Collaboration), J. Phys.: Conf. Series 340 (2012) 012048.

[18] M. Morita, Hyperf. Interact. 21 (1985) 143.

[19] J.S. Díaz, Adv. High Energy Phys. 2014 (2014) 305298.

[20] A.N. Ivanov, M. Pitschmann, and N.I. Troitskaya, Phys. Rev. D 88 (2013) 073002.

[21] M. Pitschmann et al., Phys. Rev. D 91 (2015) 074004. 